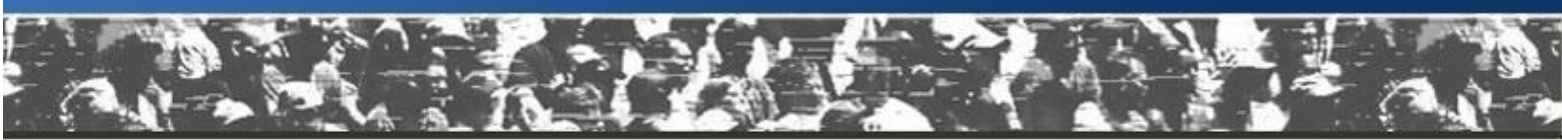

2020, Vol. 9, No.2, pp. 66-77

\title{
Teaching Environmental Anthropology in Brazil and Latvia
}

\author{
Paride Bollettin \\ Universidade Federal da Bahia - Programa de Pós-graduação em Antropologia \\ Durham University - Department of Anthropology
}

\begin{abstract}
This paper describes two courses dedicated to environmental anthropology offered respectively at the Federal University of Bahia in Brazil and the Riga Stradins University in Latvia. The courses were organized in parallel modules to enable the promotion of similar programs. The aim of this paper is to present the discussions following each topic covered by the course and the case studies chosen by enrolled students for their final works in Brazil and Latvia. The final discussion highlights how, despite the differences between the two countries, the effective engagement of students promoted the emergence of their direct participation in the course development and new ways of situating themselves in their environments.
\end{abstract}

Keywords: environmental anthropology, Brazil, Latvia, student feedback, comparative course outcomes

\section{Introduction}

Facing recent growing environmental concerns, there is an increased interest for alternative forms of relating with "nature". This is reflected in the increasing demand for courses focused on "environmental anthropology" which can be observed at academic institutions on a global level. These environmental concerns produce diverse anxieties and controversies, according to the contextual experience and the debates in diverse countries. In this panorama, this paper describes two courses of lectures focused on "environmental anthropology" which I offered in Brazil and Latvia during the first half of 2019. These courses were offered respectively at the Federal University of Bahia and Riga Stradins University. The two courses followed a very similar program, with a few adjustments due to linguistic differences and referred ethnographic examples. For this reason, a comparison of debates produced in the two cases, as well as of the topics chosen by students for their final essays, led to the emergence of a fertile comparison.

Despite the wide range of focuses and interests, environmental anthropology can be described as engaging in human- environment interrelations. Since the emergence of the discipline, anthropology has paid attention to these interrelations (i.e. Boas, 1911; Kroeber, 1939; Evans-Pritchard, 1940). A detailed review of these reflections is beyond the aims of this paper, and the reader can find a comprehensive panorama in recent publications (i.e. Haen \& Wilks, 2005; Kopnina \& Shoreman-Ouimet, 2017; Townsend, 2018). The recognition of tendencies in general core paradigms along a timeline are important to situate the courses' construction. Orlove (1908) acknowledged two alternative tendencies characterised respectively by an eco-systemic and an economicalpolitical approach. Brondizio et al. (2017, p. 11) identified a "formative period", a "specialization period", and, a contemporary "cross-disciplinary trend". It is evident that the construction of the courses needed to focus on certain tendencies to select a sample of literature that could be discussed within limited lecture hours. The choice of which topics to include in the lectures influenced the classes' dynamics, producing resonances with students' academic trajectory, as well as with their historical, social, and cultural contexts. 
The importance to reflect on the construction of the course is also related to proper anthropological practice. How can we build up courses on people-environment interrelations to produce "a deployment of the contradiction, the counterintuitive, the paradox, the rupture, as a source of methodological revelation" (Comaroff, 2010, p. 531)? Following this suggestion, the courses were aimed at producing a double and complementary intellectual exercise, for both the lecturer and students. On one side, lectures should provide an adequate knowledge to map historical and current debates on the specific topic of environmental anthropology. This aim stimulates critical thinking rather than simply enumerating historical or theoretical proposals. On the other side, lectures were constructed in order to be thought- provoking, including ethnographic examples from both "exotic" and "domestic" cases. The ambition was to let contradiction, counter-intuition, paradox, and rupture emerge from the recognition that multiple forms of entanglements with the environment share in the production of the living experience. Instead of proposing a unified idea of "nature" as a monolithic reality, the courses aimed to multiply possibilities in order for the lecturer and students to map situated experiences in original ways.

\section{The Courses}

The courses were offered during the first half of 2019 at the postgraduate program in anthropology at the Federal University of Bahia (Brazil) and at the postgraduate program in anthropology at the Riga Stradins University (Latvia). In both cases, the courses were optional so students could choose to attend or not. This meant that participants could attend the specific course according to their intellectual and research interests. This produced in both cases effective student engagement and active participation. Students' constructive attitudes during the courses generated in-class discussions and topics to be covered. The courses were developed with a common starting point and a common overall program and bibliography. However, the successive deepening of each topic followed a context-specific trajectory aligned with student feedback. I will describe below how this feedback produced intriguing results.

At the postgraduate program in anthropology at the Federal University of Bahia, the course was part of my working plan as visiting professor at the institution. This commitment, in accordance with the Brazilian academic format, included research, education, and outreach activities. For the first semester of 2019 (February to July), the program council approved my proposal for the course, under a pre-existing course name "Theories of Nature". The course had been offered for students at both masters and doctorate levels. Moreover, the course was cross-posted in the postgraduate program in history, teaching and philosophy of science in the faculty of biology at the same institution. Some vacancies were also opened to students of other academic disciplines. Fourteen students enrolled in the course from diverse backgrounds, such as, social anthropology, biology, law, history, and dance studies. The diverse student backgrounds produced animated controversies and constructive dialogues during the classes, and promoted effective interdisciplinary negotiations. The course covered a total of sixty-eight class hours, divided into seventeen lectures.

In 2018, I successfully applied for a grant for Integration of International Experience from the Boris and Ināra Teterev Foundation. In the application, after discussing it with colleagues at the Postgraduate Program in Anthropology at the Riga Stradins University, we decided to include two concentrated courses, respectively titled "Environmental Anthropology" and "Introduction to Multispecies Ethnography". These were part of a wider activity plan that included public conferences, seminars, and participation in other academic events. The courses were offered to students of the program, and a total of eleven students enrolled. They were all social anthropology students, at both masters and doctorate levels. Due to the possibility to offer parallel courses in Brazil and Latvia, I elaborated on the course's program to present the same suggestions at both institutions. The two courses offered at the Riga Stradins University have been devised as complementary in order to follow the same teaching sequence of the course offered at the Federal University of Bahia while maintaining the necessary bureaucratic division. These two courses were both composed of six lectures, for a total of twelve lectures completing twenty-four teaching hours. Despite the difference in the available time at the two institutions, the courses were planned to cover the same sequence of topics. 
At both institutions students enrolled voluntarily, making their reasons for enrolment important in understanding the value and impacts of these courses. During the first class, students of both institutions were asked to briefly introduce themselves and explain their motivations for enrolment in the course. At the University of Bahia, as mentioned above, students were from diverse postgraduate programs. At Riga Stradins University they were all from the postgraduate program in anthropology, but with undergraduate degrees in diverse disciplines (social sciences, communication, journalism, etc.). In this sense, at both institutions students presented diverse backgrounds and interests. Some students were conducting research on human-environment relations. They took the course to learn more about topics related with their investigations, and enhancing their competencies. Others declared a more "personal" motivation. For example, some expressed their interest in broadening their comprehension of how people relate with the environment in alternative socio-economicpolitical choices to hegemonic patterns. Meanwhile, others were more interested in theoretical questions covered by the courses, such as the anthropocene, post-humanism, ontological turn, etc. This polysemy reflects the multiple forms in which the environment can be approached, depending on people's perspectives. This multiplicity emerged as an intriguing stimulus for the development of collective reflections during the lectures.

\section{Topics and discussions}

In order to cover the majority of relevant discussions in both historical and contemporary environmental anthropology, the courses divided classes in equivalent teaching hours according to the requirements of each institution. The first and the second half of the "Theories of Nature" course at the Federal University of Bahia covered respectively the topics of the "Environmental Anthropology" course and the "Introduction to Multispecies Ethnography" course at Riga Stradins University. The choice of topics attempted to introduce students to the most relevant debates about people-environment interrelations, epistemological and methodological proposals, contemporary suggestions, and interdisciplinary dialogues. Lectures were organized within a participatory atmosphere, with a brief presentation of the specific topic followed by collective discussions in which students were asked to suggest examples from their experiences. In this way, each class was adapted to participants' interests rather than following a fixed script. The lecture's step-by-step program needed to be flexible in order to accommodate deviations in the interactions with and among the students. This pedagogical strategy proved to be efficient in engaging students in discussions, since the majority of them actively took part in offering examples and reflections. Moreover, it permitted theoretical, experiential, and willingness specificities of the two students' groups to emerge and to be valorised during the courses. The following paragraphs briefly outline the proposals of these topics and in-class debates.

\section{The Emergence of Nature}

During this lecture, we discussed the historical production of the concept of "nature" in current hegemonic view. We comprised ancient Greek philosophies, medieval scholars, and the influence of the modern age colonial time for the definition of "what nature is". Moreover I suggested deepening the discussion in a comparison with the alternatives in the Muslim view, to stress how diverse monotheisms produce diverse concepts of "nature". We also included a discussion of what is supposedly not included under the rubric of "nature", such as cryptozoology, in order to critically reflect on the historically-changing definitions of the natural reality. Students reacted actively to the proposal, mapping influences in their own views of the environment, and promoting various debates. At the University of Bahia, a controversy on the weight of Christian ideals of the human as "owner" of nature on the development of its modern objectification emerged. Some students developed a critique to such objectification as the core reason for current environmental problems, while others proposed, on the contrary, the suggestion that religious ethics could work as tools for promoting alternative and less exploratory practices. This discussion did not ensue at Riga Stradins University, where more attention was dedicated to a reflection of the impacts of Western views of nature on the Baltic region, which they described as being strongly influenced by historically different influences. For example, emphasis was given to the still-present "paganism" (their own definition), as an alternative approach to the local environment. The objectification of nature, in this case, was associated with the impacts of Soviet times due to its emphasis on materialism and utilitarianism. Students at both institutions recognized the necessity to reconstruct the historical emergence of the concepts as a key element to situate current experiences. 


\section{Natures and Cultures}

We analyzed the dichotomy between nature and culture along the history of anthropological debates. We started from the diverse uses of such a dichotomy as a heuristic, epistemological, and theoretical tool, and the influences of these approaches in the disciplinary debates. The lecture continued via the presentation of how the nature/culture divide worked for observing people-environment interrelations from a symbolic and a pragmatic point of view. During the expositive lecture, at Riga Stradins University, students were concerned about how to apply the dualism and binary oppositions as suggested by Lévi-Strauss. They found that the core problem was too abstract from a tangible dichotomy towards a methodological one. Interestingly, the influence that LéviStrauss has on the foundations of Brazilian anthropological tradition (Bollettin \& Athias, 2011), were also reflected in contemporary academic programs, and anthropology students at the Federal University of Bahia were used to these debates. Students from biology, law, and dance expressed their difficulties in assuming these dualisms as methodological rather than ontological. At both institutions, the inclusion of the feminist critical revision of this paradigm and the ambiguous frontier between nature and culture in genetics and in non-western people helped students to relativize this dichotomy. This topic definitively instigated a deeper reflection on two main directions: how culture and nature can be reciprocally influenced at a theoretical level, and how humans produce a sense of the world in which they live.

\section{Some Natures}

We introduced some proposals from "cultural ecology," "cultural materialism," and "ecological anthropology". The lecture started with how relations between people and their environment have been observed to illustrate the diverse forms in which societies adapt to their environments. Subsequently, the class focused the controversies between the structuralist proposal, "nature as good to think", and the materialist one, "nature as good to eat", in order to critically discuss how "nature" occupied a preeminent position in theoretical debates in anthropology, despite the diverse approaches suggested. These debates provoked animated debates at the Federal University of Bahia, due to the presence of anthropology and biology students. The first suggested the pre-eminence of a symbolic dimension of people-environment interrelations, while the second emphasized the external dimension of the environment. Such controversy was supported by direct examples from both parties: "scientific evidences" of the "reality" of nature versus multiple "meanings" for the same references among diverse populations. The exercise to map how people can materially and pragmatically relate with their environment became a chance to observe historical changes at Riga Stardins University. Here, students discussed the transformation of the Latvian environment from the Soviet time to the contemporary. They described how productive organization along time reflected social structures, from collective farms to private ones, aligned with the associated Latvian society's transformation from agricultural to urban-centred. Meanwhile, they observed how "nature" was used in political discourse during the independence from the Soviet Union, highlighting its double face. Nature appeared as both the material background influencing social production as well as the ideological milieu producing identity discourse. This topic, by the diverse feedbacks originated among the two students' groups, stressed the importance of observing pragmatic and symbolic dimensions as intermingled, an exercise greatly intriguing, especially for students with no previous experience in anthropological studies.

\section{Naturalism and Animism}

For this topic, we focused on the current developments in the study of interrelations between the environment and non-Western people. We started from Descola's redefinition of "animism" as one "mode of identification" people adopted for living with "nature", the others being "naturalism," "totemism," and "analogism." We continued introducing other concepts, such as "perspectivism" and "multi-naturalism," which further developed these ideas. In sequence, we discussed how so-called naturalism is defined in opposition to animism, and associated with Western concepts of nature. Finally, we presented possible hybridizations of these concepts, in order to critically discuss them as theoretical concepts rather than absolute models. This topic produced great interest at Riga Stradins University, where students described multiple examples of the co-presence of naturalistic and animistic modes of identification in Latvia. These co-presences highlighted how people live multiple possible interrelations with other beings, and widely with nature. What emerged is the power of this 
categorization as a heuristic tool for proliferating the diverse interspecific connections into the ethnographic experience. At the Federal University of Bahia, the debates produced a critique to the hegemonic concept of naturalism. The core interest in this case was to deconstruct a supposed unique mode of producing the objectification of nature, recognized in its materiality. Such a deconstruction has been strongly associated with the environmental concerns produced by deforestation, pollution, and other human impacts on the environment. Clearly, this lecture produced divergent and alternative impacts on students. In Latvia, it was possible to identify a more methodological interest. In Brazil, the opposition between naturalism and animism stimulated a political discussion on the best strategies for environmental preservation, and, consequently, the urgency of promoting alternative modes of relations, such as indigenous ones, to help decrease the environmental struggles in the country.

\section{Natures, Minds, and Monads}

In this lecture, we described two alternative ways of observing the interrelations between people and nature, naming Gregory Bateson's idea of "mind" and Gabriel Tarde's idea of "monad." These two concepts, both marginal in hegemonic debates, were discussed as options to review concepts of "nature" and "society." Moreover, they helped to introduce the distinction between accessing "nature" from an epistemological approach and an ontological one. The work of Gregory Bateson was mostly unknown at the two Institutions; however, the idea of "pattern that connects" as an epistemological strategy for integrating the study of multiple levels of reality fascinated both groups of students. At the Federal University of Bahia, Bateson's epistemological proposals stimulated a discussion on how to compare apparently different domains, and students from biology suggested a critical analysis of the terminology used for describing nature as proposed by students from anthropology. At Riga Stradins University, the discussion followed in the direction of deepening the understanding of how to produce such patterns, dislocating the focus from the semantics towards the alternative between the external and internal reality of nature, with clear insights from previous classes. This debate echoed in the comments on Tarde's proposal for a "monadology". While Latvian students have not had previous experience with his work, Brazilian students of anthropology did, due to his influence on a part of the contemporary anthropological debate in this country. At both institutions, the idea of "monads" having a proper active agentivity was the ground for describing the social and the natural in alternative pictures. Moving from the recognition that diverse elements, such as cells, but also companies, animals and cities, can be described as moved by imitation, invention and opposition, the debates moved toward the opposition between epistemology and ontology. In this opposition, the two groups of students recognized the core tension of the discussions about people-nature interrelations, distinguishing on one side the priority of a reality external to humans, while on the other, the possibility to integrate the two, human and non-human, in a shared collective. This topic has been described as one of the most intriguing by students of both Institutions.

\section{The Natures of Sciences}

This topic promoted the discussion of multiple "natures" assumed in scientific knowledge practices. It included an overview of various theoretical positions as well as a specific discussion of the emergence of the contemporary hegemonic idea of science. We described the proposal of seminal authors such as Bruno Latour, Donna Haraway, Stelio Marras, Arturo Escobar, David Kidner, Stefan Helmreich, Scott Atran, etc. Due to the extent of the reflections on this theme, which on its own would require an entire course of lectures, only some aspects were selected for discussion. We specifically focused on the practices of producing scientific knowledge, on the politics of science, and on interdisciplinarity in scientific discourses. Due to the specific aims of this lecture, students with a biological background at the Federal University of Bahia were very participative in offering examples, while the most critical in accepting the possibility to observe scientific knowledge practice as an object of reflection. On the contrary, anthropology students promoted the emphasis on science as a social construction, highlighting its emergence as a culturally specific form of observing the nature. Comparing the objectivity of science and of "traditional knowledge", the discussion offered the basis for a collective reflection on the efficacy of knowledge practices as key for understanding their emergence. Differently, at Riga Stradins University, students stressed the impacts of scientific knowledge in the production of a setting from which to promote a reflection on environmental politics, gender relations, and the valorisation of local knowledge. They 
observed how European Union research programs affected the scientific development of Latvia, encouraging new research fields and concomitantly creating new attitudes toward nature not only as a material resource but also as something to be conserved. Despite the divergent debates, a common point appeared in the recognition of the necessity to integrate diverse areas of specialization in interdisciplinary debates. Brazilian students described interdisciplinarity as an on-going practice in their Institution, as partially proved by this course itself, while Latvian students complained of not having the opportunity to engage in these debates.

\section{Natures' Frontiers}

This provocative topic stimulated a discussion on what can be defined as "natural" in the contemporary World. We described digital realities, exoplanetary environments, artificial intelligence and mechanical post-humanism, and cryptozoology. The beginning of the lecture portrayed how the development of a Western notion of nature was influenced by the continuative inclusion of new elements native of exotic places, as well as by the expansion of previously defined frontiers of the World. Digital and exoplanetary worlds offered the opportunity to investigate the limits between the naturalness and the sensorial experience. The tension between the bodily and mental experience of the World introduced the concept of "cyborg" and of bio-techno-social humanity. Finally, the class ended with a reflection on the possibility to assume objects as "subjects," or means with a proper agentivity, and how to do it. The discussion at the Federal University of Bahia focused in two core directions. The first went towards possible parallelisms with non-Western experiences of nature. Claiming that the limits of our experience of Nature are culturally defined, some students indicated that this constitutes a diverse reality in Afro-Brazilian religions, of which some of them are faithful. Consequently, assuming that the visible or tangible is only a part of the experience. The second focus of reflections was the technical manipulation of women's bodies as the result of gender oriented practices. The example of pregnancy medicalization was discussed as a form of manipulation of bodies' "naturalness" as a form of "artificial" construction. The manipulation of human bodies emerged as a topic of discussion also at Riga Stradins University. However, here, the reflection focused on ethical implications. This redefinition of the human body was inserted in a wider discussion on how nature is redefined in virtual environments, which enables the experience of "nature without nature" (student's sentence). Some examples referred to by students were about the urban dimension of living with artificial natural places, such as a park designed for offering the familiarity with the forest in a human-made environment. In both cases, the topic generated animated discussions on where the limits of what can be considered "natural" are situated, and on the impacts of technology on their redefinition.

\section{Human-Animal Frontiers}

This topic was offered as an introduction to the second module at the Federal University of Bahia and as the first class of the course "Introduction to multispecies ethnography" at Riga Stradins University. It focused on a panorama of the historical definition of the frontiers between the "human" and other animals. It started by presenting a brief overview of the emergence of such a frontier in philosophy, comparing the reflections of Agamben and Derrida as key guides. In sequence, we emphasised the diverse and contextual possibilities to make such a frontier flexible, according with the diverse meanings that the term "animal" can assume. Finally, current reflections on zoo- anthropologies, the "animal turn," and multispecies Worlds were introduced. The topic generated vibrant interest amongst the two groups of students. One of the key elements was the multiplicity of relations between people and other animals. In this class, it was difficult to escape the trivialization of the discussion. As appointed by Latour: "The problem with animals is that everyone has some experience with them and tons on how they resemble humans, or not" (2012: vii). The emphasis on the philosophical dimension of such a frontier worked in this direction, producing a curious attention on the specificities of each example proposed. In both cases, the domestic, intimate dimension of people's interrelations with animals (pets), and the consequent anthropomorphism, was compared with their objectivity in industrial farms as well as in public policy. The introduction of alternative non-Western concepts of animality and humanity, already discussed in previous classes, helped to make the panorama more complex. Both in Brazil and in Latvia, students were interested in investigating such ambiguous frontiers in detail. In the first case, some students from anthropology emphasised how these frontiers depend on cultural backgrounds, citing examples from Indigenous and Afro-Brazilian peoples. Meanwhile, some students from biology argued in support of a 
more "objective" distinction based in morphogenetic human singularity. These debates provided the backdrop for the following classes. In the second case, students at Riga highlighted the historical changes in daily experiences of these frontiers. A comparison between the Soviet time and the post-Soviet time offered an opportunity to discuss how the urbanization of Latvia led to a redefinition of these frontiers, with the emergence of "abstract animals", such as in zoological parks or in media, as substitutes of "companion animals", such as the one people experience in rural life (student's definition). The transformation of these frontiers here was associated with the social, political, and economic life of people. It is evident how such a broad topic generated alternative discussions, promoting the emergence of differentiated approaches to the topic amongst the two groups of students.

\section{Wild and Domesticated}

This class focused on another ambiguous and controversial dichotomy: the concepts of "wild" and "domesticated." We retraced the development of this dichotomy in diverse disciplines: anthropology, archaeology, biology, etc. From this panorama, which was easily identified as anthropocentric by students at both Federal University of Bahia and Riga Stradins University, we moved towards current discussions about the bio-social co-domestication of humans and other-than-humans. Finally, we discussed the multiple implications of this dichotomy in social, economic, political and ethical dimensions. At Riga Stradins University, students reflected on the insertion of Latvia in European Union environmental politics, suggesting examples of "rewilding" policies implemented in the country. They discussed how these policies promoted a renewal of ideas about "nature" while suggesting that other-than-human beings act in this redefinition. At Federal University of Bahia, the discussion moved towards two complementary directions. On one hand, students focused on the social implications of this dichotomy. Citing examples from the controversies between environmental protection and "traditional communities," they emphasised the contraposition between wild and domesticated as related to power structures. They suggested that this dichotomy is grounded on the marginalization of nature as well as of traditional communities. Meanwhile, some students, especially from biology, promoted a reflection on the intermingling redefinition of the multispecies collectives describing multispecies co-evolution. In both cases, the class stimulated a reflection on the bio-social dynamics embedded in "domestication" processes, with the inclusion of other-than-humans as proper subjects of these processes.

\section{Biopolitical Beings}

This class focused on the concept of "animal biopolitics." The first part of the class introduced the concept of "biopolitic" as it was introduced by Foucault and successively revisited by other scholars. After the introduction, the class described how this concept can be useful in describing a range of human-other-than-human collectives: from legal management of other-than-human species, to animal rights, animal capital, and biogenetic manipulation. This class emphasized how the other-than-human body, whether a vegetable, animal, or simplecelled organism, is the arena of a political game in which concepts of "nature" are mobilized to organize interspecific relations. At the Federal University of Bahia, students suggested the historical transformations of northeast Brazil as an example of the biopolitical influences in the production of the environment, with an introduction of "exotic species" in place of "native" ones. As a consequence, also with the stimulus of students from biology, the definition of protected areas emerged: protection referring to "isolating some species from anthropogenic influences" (student's definition). Meanwhile students recognised that such "isolation" is only a chimera, due to the pervasiveness of anthropogenic influences on the environment. At Riga Stradins University, the debate turned to the emergence of new concepts of animal rights. Students highlighted the difference between the treatment of diverse species - some are bred to die while other are compared with humans (pets). The biopolitic of life emerged as preeminent in the definition of social, economic, and political dimensions of multispecies collectives. Meanwhile, the current debate about "invasive species" was an example of the management of life according with specific concepts aimed at describing interspecific conflicts. 


\section{Cultural beings}

This class introduced the diverse forms of conceiving other-than-humans as having a proper "culture." It started with a panorama of alternative definitions of "culture" in anthropology, indicating toward the polysemy of the concept. Two complementary questions were presented in sequence. The first focused on how it would be possible to define other-than-human culture, and if this should present similarities or radical differences from the human culture to be identified. The second promoted a reflection on the epistemological productivity of the concept of other-than-human culture for the implementation of interdisciplinary dialogues on multispecies collectives. At the Federal University of Bahia, the following discussion grew from the alternative approaches to other-than-human culture described in previous classes. The recognition that culture is shared property between both humans and other-than-humans among Amerindians promoted the necessity of situating the question culturally. Moreover, the interface between anthropology and biology was described as a heuristic tool to achieve cultural life beyond the human. In this perspective, culture was identified as shared property of all beings, also from a biological approach. This promoted a reflection on the notable difference between situating culture's emergence from a biological or social background. At Riga Stradins University, the question stimulated a discussion on the limits of our comprehension of possible other- than-human cultures. According to the diverse definitions one can adopt, some other-than-humans would be included or excluded. Students highlighted how this ambiguity can promote anthropomorphism of other beings or, alternatively, the recognition of the existence of different cultures in diverse species. Meanwhile, the possible cultural life of other-than-humans was described as common among people living in the countryside, supporting the affirmation that a close familiarity with other-than-humans can promote their culture's recognition.

\section{Intermingled Beings}

The aim of this class was to describe how diverse beings can assume diverse identities according to their relational networks. It started with a description of current discussions about the "anthropocene" as a panorama in which to situate critically the pre-eminence of the human in our epistemic-ontological approach to multispecies collectives. In sequence, we discussed how it is possible to recognize the reciprocal influence of diverse beings in the common production of shared collectives. We specially focused in detailing how otherthan-humans affect voluntarily or involuntarily humans on macroscopic or microscopic levels. From the recognition of the common becoming in which human and other-than-humans are merged, the class promoted a reflection on the multispecies approach as a response to current environmental struggles. At the Federal University of Bahia, students stimulated a discussion between the effective agentivity of other-than-humans, such as an endangered species as an actor in the definition of environmental protection policies, or of pets in modifying peoples' daily life. Depending on the recognition of such effectiveness, and of its voluntariness, the interrelations between diverse beings has been described as assuming alternative epistemic-ontological dimensions. Meanwhile, this discussion reflected the necessity to integrate complementary disciplinary knowledge, integrating anthropological and biological sciences. At Riga Stradins University, the topic stimulated a reflection on the current environmental degradation affecting several ecosystems. On one side, some students suggested that capitalistic ideology, promoting the objectification of other-than-humans, can be observed as the main cause of environmental disasters. Capitalism was opposed to "traditional" interrelations with the environment, in which people were used to experience a more intimate contact with other-than-humans. Meanwhile, the discussion also focused on how to map such multispecies interconnections, in which the biological, the social, and the technical emerged as component aspects.

\section{Final Essays}

At the end of the course, students at both the Federal University of Bahia and Riga Stradins University were asked to produce a final paper. They were instructed to choose a case on their own, using the discussions and bibliographical materials of the course to produce a brief ethnographic description. The format of the final essay at the two institutions was identical. The required text length was ten pages, with a minimum amount of ten bibliographical resources from the course. Students could also use more than ten sources and incorporate bibliographical sources not used in the course. The aim of this final essay was not only to evaluate the effective 
appropriation of the course contents, but also to stimulate their autonomous application in specific, concrete case studies. Students were informed about this task at the beginning of the course so they had ample time to identify the ethnographic case of their interest. Some students decided the focus of their essays according to their on-going research activities (for example some specific element of their ethnography for their Master or $\mathrm{PhD}$ dissertations), while others decided to focus on a new case unrelated to previous research experience.

The elaboration of a final essay based on direct field experience of students responds to a peculiarity of ethnographic enquiry. Direct participation and observation, even if limited to a short time period as in this case, constitutes the core methodological tool of anthropological enquiry. The realization of a final essay based on direct observation enabled students to develop in-depth knowledge of what Fassin defined "[the place] where true life and real lives meet" (in Joshi, 2014). Students were also required to auto-evaluate their efforts and the level of their appropriation of the course contents. Despite the fact that students were not requested to produce a prolonged field experience, the possibility to directly investigate a concrete case through which to discuss bibliographical materials and in-class discussion constituted an exemplar opportunity to critically reflect on the course. Moreover, first-hand experience offered students the chance to elucidate the topics which stimulated their curiosity and attention, since fieldwork is the tentative "to understand another life world using the self - as much of it as possible - as the instrument of knowing" (Ortner, 1995, p. 173). Therefore, the elaboration of final essays responded to a double aim. On one hand, it enabled students to directly apply what they learned during the course to the study of a concrete case, not only as a theoretical and abstract discussion. On the other hand, their choices for the final essays allowed them to describe what most attracted their interests. The wide range of chosen topics illustrates the students' sensibilities with the core theme of people-environment interrelations.

At the Federal University of Bahia, students' essay topics included: how gender dimension affects the idea of naturalness of women's bodies in biomedical research, how the Law (understood as a system of values) reflects the dichotomy between nature and culture, how childbirth reflects conceptions about a woman's body in medical and alternative practices, how athletic practices on a beach in Bahia present imaginary about what is natural and cultural, how criminal reports during colonial Brazil show the idea of a naturalness of slaves' bodies, how the revision of the nature/culture dichotomy can promote alternative dance performances, how fishermen on a village of Bahia's coastline manage the environment in a changing social organization, how archaeological research in a Brazilian National Park highlight specific ideas about human manipulation of the local environment, how the agro-ecological movement in Bahia is defined by biopolitical multispecies relations, and how the Brazilian cordialidade (cordiality) can be observed as the naturalization of hybrid communities.

At Riga Stradins University, student essay topics included: how Latvian media reported current climate change effects, how the construction of a new port changed people-environment relations in a riverside village, how Latvian media presents wildlife in local and national newspapers, how bathing house traditions echo specific ideas about the naturalness of the body, how the zero-waste movement in Latvia redefined people's sensibility with their environment, how a Latvian movement around new forms of living in urban spaces promoted alternative ideas on people-environment relations; how other-than-human animals' subjectivities are presented in Latvian traditional literature and in children imaginaries, how the frontier of what is natural has been redefined in a digital security platform of a Latvian bank, how farmers in Latvia produce multiple relations with other-thanhuman wild and domestic animals, how invisible beings are presented in traditional Latvian literature as proper subjects, how the Latvian Fund for Nature sensitizes people to the local environment.

It is clear how the possibility to choose their own "field" enabled students at both institutions to deepen some case studies with which they were already familiar. Their choices also indicate which topics discussed along the course most stimulated their interest. In this sense, this modality to realize the final essays appears to be adequate in achieving a double finality. It offers a significant chance for students to appropriate course contents in a practical way, adapting them to the study of a concrete case. The following self-evaluation contributed to this by allowing them to observe which point of their analysis should be further developed and which ethnographic details were most relevant for the overall project. The indication of bibliographical sources was helpful for the analysis in some cases, as well as the request for more detailed descriptions in other cases. Self-evaluation promoted their reflection on the importance of thinking ethnographically about the learning process. This 
stimulated their attentiveness to the process of incorporating the course contents as a form of reflection on both the case studies and on their own relations with the theme of the course. The high level of the students' final essays at both Institutions promoted the organization of two parallel collective books, each one collecting a selection of these paper after a double blind peer-review process. The Editorial Cooperative of the University of Padua (Italy) will publish these books in 2020.

\section{Conclusion: some notes on teaching Environmental Anthropology}

The topics discussed by students in their final essays let them observe their main interests related with the course. A learning process based on the ethnographic practice instead of the emphasis of theory (Da Col \& Graeber, 2011), is also reflected in the emphasis dedicated to students' participation in the discussions. These discussions were aimed not only at debating the topic of each class, but also at stimulating their reflections on the fields they would later analyze for their essays. The persistent requirement of students to draw parallels during the discussions was aimed at a continuative process of reflection on how to join theoretical reflections with specific examples of their own. This strategy offered students a background in which to situate their final work, and let their interests emerge unceasingly.

What appears from the list of case studies chosen for the final essays is the complex panorama of intriguing possibilities offered by a course about "environmental anthropology." The ambiguity of the theme, as well as its necessary resonance with the students' sensibilities", defined a teaching-learning environment that promoted an incessant redefinition of what is understood by "environment" and "anthropology."

As enumerated above, some students decided to discuss field cases in which people directly related with their surrounding environment, such as in the case of the fishing village in Bahia or the agricultural multispecies collectives in Latvia. Others decided to analyze how people use concepts of "nature" and "culture" in their own daily lives as toolkits for the production of social relations, such as in the case of sport practices on the beach in Bahia and the bathing houses in Latvia. Others decided to delve into how the nature-culture dichotomy is presented in official discourses, such as the case of slaves' representation as "natural bodies" in colonial Brazil and of the media's presentation of nature in Latvia. Others, finally, decided to engage in examples in which naturalness is the key for political activism, such the representation of women's body as natural in biomedicine in Bahia or the zero-waste movement in Latvia. In all of these papers, students defined what is the "environment," what is "nature," and what is "culture," in diverse and sometime contradictory ways, according to their own sensibilities.

The same indications are also offered by an overview of the collective discussions during the classes. A wide range of interests emerged as connected with the topics chosen for each lecture. Some of these were more "classical," such as a reflection on the differences between so-called animistic and naturalistic societies, or between capitalism and traditional societies. Other were more provocative, such as the controversies about other-than-humans as subjects of rights, or about the scientific bio-social production of "naturalness." Interestingly, despite the obvious differences emerging from the two socio-historic-academic environments, from the two epistemic-ontological traditions, and from the specific interests of enrolled students, the participants oscillated between the diverse possibilities offered by the courses' contents in both Institutions. Moreover, the active engagement of students in these discussions enabled them to participate directly in the educational objective (Kopnina, 2016). Meanwhile, the possibility to develop effective interdisciplinary dialogues via the participation of students with diverse backgrounds - at the Federal University of Bahia students were enrolled in diverse courses while at Riga Stradins University they had different undergraduate trajectories responded to the current inescapability of such dialogues once we recognize that people and their environments are merged in a bio-social becoming (Ingold \& Palsson, 2013).

Some notes should be appointed for future courses on the topic of environmental anthropology. Due to the short course time, at Riga Stradins University I did not plan outdoor teaching. At Federal University of Bahia, one class, the one dedicated to the topic "Wild and Domesticated", was offered at the Salvador Zoological Garden. This experience of outdoor teaching was successful in engaging students in a direct experience, due to the presence of animals in cages and outside of the cages. The realization of further outdoor classes in future 
courses would be useful to promote more direct and personal experiences. Also the required field exercise for the final essay worked in this direction, and certainly should be maintained as a pedagogical and personal task. During the classes I used no visual supports, such as films or pictures, emphasizing students' participation through engagement in debates. The inclusion of these supports in future courses could be tested in order to check its efficiency in students' reflections about specific topics. Finally, a major indication produced by the courses I described is the necessity to promote a deeper interdisciplinary dialogue. This emerged as a question during the debates, since several of the covered topics are studied from diverse disciplinary perspectives. In order to achieve this result, a strategy could be to include works from other disciplines in the bibliography.

How do people experience the environment? How are these experiences shaped by humans and other-thanhumans? How do social, political and ideological forces model these interrelations? All of these questions arose during the courses, both in collective discussions and in the students' final essays. In this sense, the courses offered a panorama of current developments in environmental anthropology, while promoting the critical engagement of students with their own experience of the frontiers between humans and other-than-humans, and what is defined as "nature" and "culture." What emerges from the courses in Brazil and Latvia is the powerfulness of an environmental anthropology course for stimulating students' reflections on their personal engagement with the environment. Anthropology should promote curiosity towards contextual knowledge rather than offering an instrumental toolkit (Strathern, 2006). Students' engagement with the courses demonstrates the effectiveness of this claim. As described by some students after the end of the courses in private emails, what they learned was not only the complex development of a sub- discipline and the melding of diverse disciplinary perspectives. They also observed their position in a wider panorama in which other-than-human social beings share their lives with humans in which intermingled social, cultural, and political forces produce complex multispecies collectives.

\section{References}

Boas, F. (1911). The Mind of Primitive Man, New York: Macmillan.

Bollettin, P., \& Athias, R. (eds.) (2011). Lévi-Strauss visto dal Brasile, Padova: Cooperativa Libraria Università di Padova.

Brondizio, E., Adams, R.T., \& Fiorini, S. (2017). History and scope of environmental anthropology. In: H. Kopnina and E. Shoreman-Ouimet (eds.), Routledge Handbook of Environmental Anthropology, London: Routledge, pp. 10-30.

Comaroff, J. (2010). The end of Anthropology, Again: On the Future of an In/Discipline. American Anthropologist, 112, 4, 524-538.

da Col, G., \& Graeber, D. (2011). Foreword: The Return of Ethnographic Theory. HAU: Journal of Ethnographic Theory, 1, 1, pp. vi-xxxv.

Evans-Pritchard E. (1940). The Nuer: a description of the modes of livehood and political institutions of a Nilotic people. Oxford: Claredon Press.

Haen, N., \& Wilks, R (eds.) (2005). The Environment in Anthropology: A Reader in Ecology, Culture, and Sustainable Living. New York: New York University Press.

Ingold, T. \& Palsson, G. (Eds.) (2013). Biosocial Becomings: Integrating Social and Biological Anthropology, Cambridge: Cambridge University Press.

Joshi, M. (02/2014). AE interviews Didier Fassin (Institute for Advanced Study, Princeton, NJ). Retrieved on September 2019 from: https://www.americanethnologist.org/features/interviews/ae-interviews-didier-fassininstitute-for-advanced-study-princeton-nj 
Kopnina, H., \& Shoreman-Ouimet, E. (eds) (2017). Routledge Handbook of Environmental Anthropology, London: Routledge.

Kopnina, H. (2016), 'Of Big Hegemonies and Little Tigers: Ecocentrism and Environmental Justice', The Journal of Environmental Education, 47(2): 139-150.

Kroeber A.L. (1939). Cultural and Natural Areas of Native North America. Berkeley: University of California Press.

Latour, B. (2012). Foreword: The Scientific Fables of an Empirical La Fontaine, In: V. Despret, What Would Animals Say if We Asked the Right Questions?, Minneapolis: University of Minnesota Press, pp. vii-xiv.

Orlove, B. (1980). Ecological Anthropology. Annual Review of Anthropology, 9, 235-273.

Ortner, S. (1995). Resistance and the Problem of Ethnographic Refusal. Comparative Studies in Society and History, 37, 1, 173-193.

Strathern, M. (2006). Useful Knowledge', Proceedings of the British Academy, 139: 73-109.

Townsend, P.K. (2018). Environmental Anthropology: From Pigs to Policies. Long Grove, IL: Waveland Press. 